expression was significantly increased in tumours compared to adjacent normal tissue (figure 1A). Mice treated with CD5-2 + anti-PD1 antibody had significantly smaller tumours $(50 \%$ reduction) compared to mice treated with either agent alone, controls, or untreated mice (figure 1B). Histologically, tumours in the CD5-2 + anti-PD1 group exhibited a more favourable immune infiltrate (significantly higher CD3 + and CD8 $+\mathrm{T}$ cells and lower Ly6G+ neutrophils) compared to tumours in other groups (figure 1C). Tumours in CD5-2-treated mice had less leaky vasculature (as measured by Dextran beads extravasation) and less tumour hypoxia (carbonic anhydrase IX staining) compared to non-CD5-2-treated mice (figure 1D).

Conclusions In the DEN model, CD5-2 normalised tumour vasculature and reduced tumour hypoxia. CD5-2 plus antiPD1 antibody reduced tumour size possibly by altering immune infiltrate to being immunosupportive. The combination of vascular normalisation by targetting VE-Cadherin and immunotherapy is a promising novel approach to treat HCC.

\section{IDDF2020-ABS-0213 CXCR2 BLOCKADE DISRUPTS TUMOR TRAFFICKING OF MDSC TO POTENTIATE IMMUNOTHERAPY EFFICACY}

${ }^{1}$ Tsz Tung Kwong*, ${ }^{1}$ Chi Hang Wong, ${ }^{2}$ Yu Feng, ${ }^{2}$ Zhe Wen Xiong, ${ }^{2}$ Jing Ying Zhou, ${ }^{3}$ Alfred Sze Lok Cheng. 'Department of Clinical Oncology, Faculty of Medicine, the Chinese University of Hong Kong, Hong Kong; ${ }^{2}$ School of Biomedical Sciences, the Chinese University of Hong Kong, Hong Kong; ${ }^{3}$ School of Biomedical Sciences; State Key Laboratory of Digestive Disease, the Chinese University of Hong Kong, Hong Kong

\subsection{6/gutjin-2020-IDDF.5}

Background The heterogeneity and diverse pathogenic conditions of HCC construct an immunosuppressive tumor microenvironment (TME) which may lead to low immune checkpoint blockade (ICB) therapeutic responsiveness. Therefore, alleviating immunosuppression to potentiate ICB anti-cancer immunity is in urgent need. Myeloid-derived suppressor cells (MDSCs) with potent $\mathrm{T}$ cell suppressive property are correlated with poor prognosis and unsatisfactory ICB response in HCC. In this study, we aimed to study the potential efficacy and functional mechanisms of targeting C-X-C motif chemokine receptor 2 (CXCR2) chemotaxis pathway to block MDSC tumor infiltration, enhancing ICB efficacy using preclinical orthotopic HCC mouse models.

Methods CXCR2-chemotaxis pathway activation in MDSCs was determined by multi-color flow cytometry in tumor and paired non-tumor liver specimens from HCC patients, as well as healthy blood samples. Therapeutic efficacy of CXCR2 blockade was conducted in an orthotopic mouse model using AZD5069 (100 or $150 \mathrm{mg} / \mathrm{kg}$ ) which is a CXCR2 antagonist currently undergoing clinical trials and in combination with anti-PD-L1 antibody (10F.9G2). Tumorigenic monitor, immune profiling and survival analysis were performed. Mechanistic study was determined using lentivirus-based gene knockdown in human-blood cell models.

Results The result showed that both monocytic (M-MDSC) and polymorphonuclear (PMN-MDSC) populations are elevated in HCC liver tissue compared to healthy donor (HD) control. CXCR2 was widely expressed in immune cells, in particular for MDSC, while its ligand interleukin-8 (IL-8) was expressed in the majority of tumor cells, as well as CD $45^{+}$ leukocytes in HCC. Notably, the blockade of CXCR2 chemotaxis pathway significantly inhibits MDSC trafficking into tumor microenvironment in HCC orthotopic mouse model.
Furthermore, co-blockade of CXCR2 and PD-L1 remarkably reduced tumor weight when compared to a single treatment, in which intratumoral $\mathrm{CXCR} 2^{+} \mathrm{PD}-\mathrm{L} 1^{+}$MDSC was positively associated with tumor burden.

Conclusions Our data demonstrated the intricate link between IL-8/CXCR2 axis and MDSC trafficking to TME, providing insight into the immunosuppression mechanism in HCC. Targeting IL-8/CXCR2 chemotaxis pathway may potentiate ICB responsiveness, serving as a novel potential therapeutic option for effectively combined immunotherapy in liver cancer.

\section{Clinical gastroenterology}

\section{IDDF2020-ABS-0033 THE EFFECT OF IMMUNOMODULATORS AND OTHER FACTORS ON THE PERSISTENCE OF BIOLOGICAL AGENTS FOR CROHN'S DISEASE AND ULCERATIVE COLITIS: DATA FROM THE AUSTRALIAN POPULATION-BASED REGISTRY}

Yanna Ko*, Rupert Leong, Sudarshan Paramsothy. Concord Repatriation General Hospital, Sydney, Australia

\subsection{6/gutjnl-2020-IDDF.6}

Background Treatment persistence (duration of medication use) provides real-world evidence on therapeutic effectiveness, tolerability and prescriber and patient preferences. Biological agent persistence in Crohn's disease (CD) and ulcerative colitis (UC) was compared from the national population-based registry with no hierarchical prescribing order. We hypothesized immunotherapy co-therapy would increase persistence through decreased immunogenicity.

Methods A randomly selected ten percent subgroup of the prospectively collected population-based registry from the Australian Pharmaceutical Benefits Scheme between June 2005June 2019 was analysed. Treatment persistence of adalimumab (ADA), infliximab (IFX), vedolizumab (VDZ) and ustekinumab (UST) was compared.

Results 2499 patients were included consisting of 3713 lines of therapy (2864 CD, 849 UC) which equated to 7470 person-years of follow-up. In CD, UST had the highest overall persistence rate (median persistence rate $>74.6 \%$ where 24.6 months is the maximum follow up time recorded), followed by VDZ, IFX and ADA ( $p=0.03)$ (figure 1$)$. In UC, VDZ had

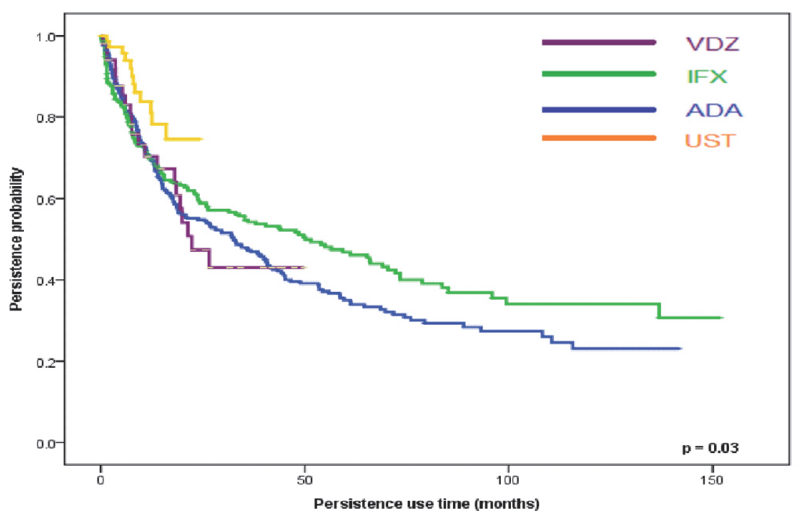

Abstract IDDF2020-ABS-0033 Figure 1 Persistence Kaplan meier graphs- Luminal crohn's disease persistence 
the highest overall persistence rate (median persistence rate $>50.3 \%$ where 47.4 months is the maximum follow up time recorded), followed by IFX and ADA (p <0.001) (figure 2).

Biological agent persistence correlated with immunomodulator co-therapy in $\mathrm{CD}\left(\mathrm{R}^{2}: 0.65 \mathrm{p}<0.001\right)$ and $\mathrm{UC}\left(\mathrm{R}^{2}: 0.50\right.$ $\mathrm{p}<0.001)$. Thiopurine co-therapy increased persistence for IFX and ADA in CD and UC $(\mathrm{p}<0.001)$ with a trend for higher persistence for UST in CD $(\mathrm{p}=0.06)$ (figures 3,4). Methotrexate decreased persistence for VDZ in UC and had no effects on IFX and ADA in UC and CD.

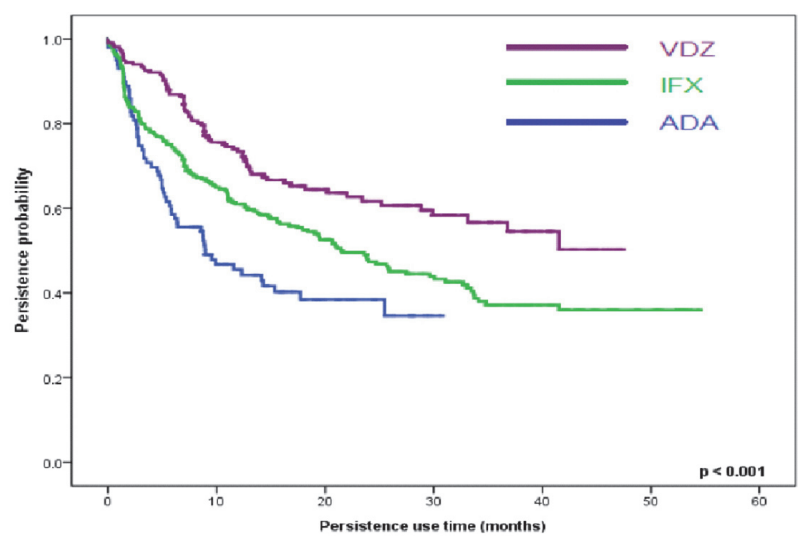

Abstract IDDF2020-ABS-0033 Figure 2 Persistence Kaplan meier graphs- Moderate-severe ulcerative colitis persistence

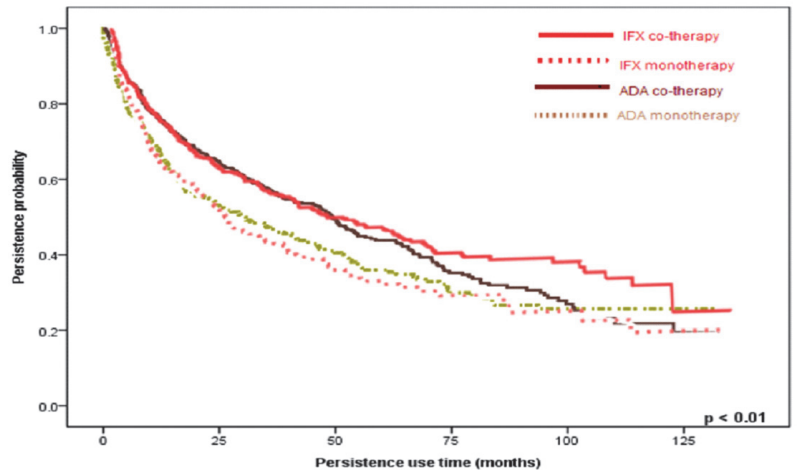

Abstract IDDF2020-ABS-0033 Figures 3 Persistence Kaplan meier graphs-Thiopurine co-therapy in Crohn's disease

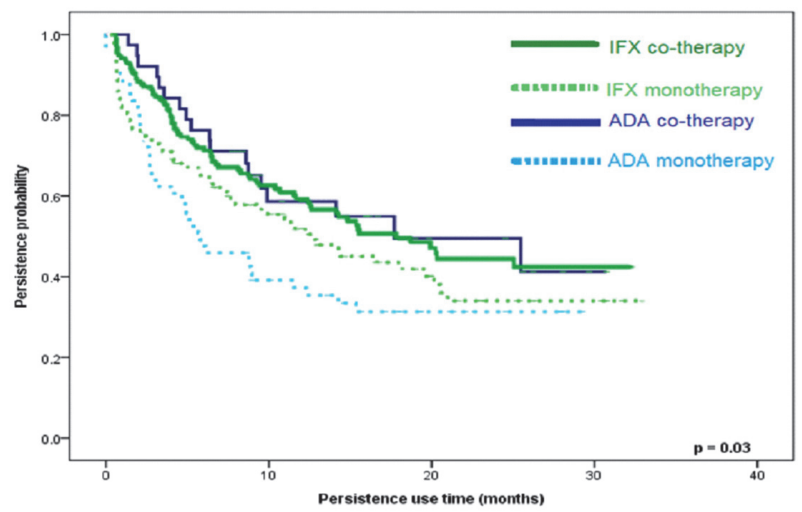

Abstract IDDF2020-ABS-0033 Figures 4 Persistence Kaplan meier graphs-Thiopurine co-therapy in Ulcerative colitis
On multivariate analysis, higher persistence was seen in males (HR: 0.75 95\%CI: 0.59-0.94) and with immunomodulator co-therapy (HR 0.77 95\% CI: 0.61-0.97, $\mathrm{p}=0.03$ ).

Conclusions This real-world data reflecting treatment effectiveness, tolerability and patient and prescriber preferences supports the use of ustekinumab and vedolizumab over antiTNF agents in CD and UC respectively to achieve high treatment persistence, possibly secondary to low immunogenicity. It also highlights the substantial increase in biological therapy persistence with thiopurine co-therapy, rather than methotrexate.

\section{IDDF2020-ABS-0165 ACCURACY AND APPLICABILITY OF THE ARTIFICIAL INTELLIGENCE INTEGRATED SOFTWARE IN Z-LINE SEGMENTATION}

${ }^{1} \mathrm{Hang}$ Dao*, ${ }^{2}$ Hung Le, ${ }^{1}$ Binh Nguyen, ${ }^{1} \mathrm{Hung}$ Nguyen, ${ }^{3} \mathrm{Huy}$ Manh, ${ }^{3} \mathrm{Kien} \mathrm{Ha},{ }^{3} \mathrm{Hai} \mathrm{Vu}$. ${ }^{1}$ Institute of Gastroenterology and Hepatology, Vietnam; ${ }^{2}$ Hanoi Medical University hospital, Vietnam; ${ }^{3}$ Hanoi University of Science and Technology, Vietnam

\subsection{6/gutjnl-2020-IDDF.7}

Background Artificial intelligence (AI) and its applications in developing software for assisting endoscopic education are novel research directions around the world in general and in Vietnam particularly. This study aims to assess the software accuracy in assisting Z-line segmentation by comparing with medical doctors' detection results and record doctors' satisfaction in scale, time-of-implementation in interactive mode, and integrated mode.

Methods This research was conducted from April 2019 to July 2020. For the development of the Z-line detection algorithm, a dataset of 533 high-definition endoscopic WLI (white-light) images in diverse forms of Z-line were collected. Preliminary assessment of the AI algorithm yielded a good IoU (Interception over Union) of $72 \%$. The software was subsequently developed in 4 modes, including manual mode, interactive mode (using Superpixels-BPT), automatic mode (using AI algorithm), and integrated mode (the combination of BPT and U-Net). 30 endoscopic images were assigned to 2 groups of doctors (naive and experienced group) for the Z-line detection using the software in 4 modes. Assessment indicators including time-of-implementation, number of mouse clicks, satisfaction in scales, and IoU metric with expert's ground-truth were taken into account.

Results The IoU metric of interactive and integrated modes in the experimental dataset deviated from $87.3 \%$ to $88.5 \%$ with no statistical difference to the IoU value of manual mode, and mean IoU metrics from the results of 2 doctor groups were over $85 \%$. The mean values of time-of-implementation in interactive mode and integrated mode were not statistically different from manual mode. The median number of mouse clicks each use in the interactive mode and the integrated mode were 24.5 and 15.5 times, respectively. The software received good feedbacks from the doctors, with the mean values of satisfaction scores of automatic mode, interactive mode and integrated mode were 7.19, 7.26, and 7.18, respectively.

Conclusions The development of the software for detecting endoscopic anatomy landmarks is a novel and feasible research direction in Vietnam. Further studies could focus on detecting some specific lesions. 